\title{
Some influences of accent structure on melody recognition
}

\author{
MARI RIESS JONES and JACQUELINE T. RALSTON \\ Ohio State University, Columbus, Ohio
}

\begin{abstract}
Two experiments were carried out to investigate the roles of joint accent structure and familiarity in delayed recognition of relatively long tonal melodies. Melodic themes of target melodies were defined by correlating contour-related pitch accents with temporal accents (accent coupling) during an initial familiarization phase. Later, subjects gave recognition responses to key-transposed versions of the target melodies as well as to decoys with same and different contour accent patterns. In Experiment 1, all to-be-recognized melodies occurred both in an original rhythm, which preserved accent coupling, and in a new rhythm, which did not. Listeners were best at distinguishing targets from different decoys, especially in the original rhythm. In Experiment 2, the familiarity of target tunes and the rhythmic similarity in recognition were varied. Similar rhythms preserved accent coupling, whereas dissimilar rhythms did not. Listeners were most adept in distinguishing familiar targets from different decoys (Experiment 2A), particularly when they appeared in novel but similar rhythms. However, in similar rhythm conditions, listeners also frequently mistook same decoys for targets. With less familiar targets (Experiment 2B), these effects were attenuated, and performance showed general effects of pitch contour.
\end{abstract}

In this article, we will examine the average listener's ability to recognize melodic themes and variations in music as a function of rhythmic context. The issue of memory for melody is not unrelated to memory for other kinds of material, such as ballads, poems, and TV jingles (see, e.g., Wallace \& Rubin, in press). All have some claim to establishing in listeners a dynamic structure that influences later recognition. In the present research, we have explored certain relationships between the form of an original event and that of its dynamic reinstatement.

In musical compositions, composers commonly announce a melodic theme and follow it with several variations, thereby evoking different levels of recognition in listeners. Indeed, whereas a psychologist may wonder what degrades melodic recognizability, composers seem to know this intuitively and capitalize on it to evoke different degrees of thematic clarity. Their techniques yield variations that are based on rearrangements of both melodic (tonal) and rhythmic (temporal) elements of the original event. In the present research, we rely on simple definitions of melodic and rhythmic parameters to explore their impact on melody recognition. Our general goal is to in-

This research was supported by Grant BNS-8204811 from the National Science Foundation awarded to Mari Riess Jones. The authors thank Konnie Toth for data collection and preliminary analyses, as well as Susan Holleran, Caroline Palmer, Caroline Monahan, and an anonymous reviewer, for helpful comments on earlier versions of the article. Special thanks are due to Mitch Pratt, who conducted a portion of this work as part of a Master's degree requirement. Correspondence concerning this article should be addressed to Mari Riess Jones, Department of Psychology, 142 Townshend Hall, Ohio State University, 1885 Neil Avenue, Columbus, $\mathrm{OH} 43210-1222$. vestigate ways in which different combinations of melodic and rhythmic accent patterns evoke a recognition response of some earlier theme.

\section{Background}

In tonal music, a melody is a particular serial arrangement of pitch intervals that also establishes a sense of key. Pitch intervals, measured in logarithmic units (semitones, ST), are the pitch distances between successive frequencies. ${ }^{1}$ Variations of a melody often involve systematic changes of these intervals or of the pitches themselves. For instance, a uniform change of all pitches to higher or lower frequencies that preserves both a melody's serial order and its pitch interval properties is a variation in which the theme is easily recognized (see, e.g., Burbidge \& Jones, 1982; Dowling \& Harwood, 1986). Even some serial rearrangements of pitches that preserve relatively few adjacent pitch intervals still move listeners to "hear" the original theme, presumably because these rearrangements capture attention-getting aspects of the original melody. One such variation that will be of interest is that which preserves a melody's contour, namely its "ups" and "downs" in pitch (Dowling, 1978; Dowling \& Fujitani, 1971; Dyson \& Watkins, 1984; Edworthy, 1985; Jones, Summerell, \& Marshburn, 1987; Watkins, 1985). At least with short unfamiliar sequences, invariances in pitch contour have a pronounced influence on melody recognition, particularly after brief retention intervals (Bartlett \& Dowling, 1980; Dowling \& Bartlett, 1981; Dowling \& Harwood, 1986). One reason why pitch contour has such powerful effects may be that contour itself offers salient accents that are based on directional pitch changes (Boltz \& Jones, 1986; Thomassen, 1982). 
The fact that any melody is a temporal arrangement of pitches underscores that its structure depends fundamentally on relationships of pitches in time. Relative timing refers to the time of one event, taken with reference to the time of another, and it is variously expressed (e.g., as ordinal, interval, or ratio time relations) (Jones, 1976). Relative timing among different pitches is an integral part of a melody's structure, in which timed pitches can occupy adjacent or nonadjacent serial locations. The fact that a melody's pitch contour can be psychologically compelling attests to the import of time relations among nonadjacent events, because usually the pitches that mark contour changes are nonadjacent events.

This raises the possibility that people attend selectively in time to nonadjacent events (Jones, 1976). For instance, pitches at contour change points may attract more attention and ultimately form a more memorable, higher order melodic theme for listeners. If so, time relations among these pitches should govern "when" people attend, and hence "what" they remember (Jones \& Boltz, 1989).

In the present analysis, it is assumed that pitch contour contributes to a psychologically salient accent pattern. At least some recent work on pitch accenting questions this assumption (Deliege, 1987). However, in this and in related work that casts doubt on the general salience of pitch relationships, relatively short and/or isochronous tunes have been employed (see, e.g., Monahan \& Carterette, 1985 ). Since pitch accents are often associated with nonadjacent events, it is possible that their influence will be more evident in longer tunes. Relatively long, rhythmically patterned tonal melodies were the stimuli in the present delayed recognition task. We varied the extent to which a to-be-recognized tune preserved certain time relations among contour-related pitch accents of previously presented melodies. In recognition, these time relationships were preserved among all pitches of a melody, among only certain nonadjacent accented pitches, or among neither adjacent nor nonadjacent pitches. In an attempt to influence listeners' attention to these pitch accent points, we also varied the rhythm in which to-be-recognized melodies occurred, so that the pitch theme carried by nonadjacent notes was either selectively highlighted temporally or not.

The design is identical to that of Jones et al. (1987). In recognition, listeners attempted to differentiate previously presented targets from decoys with same and different contours. Each target melody and its associated decoys occurred either in an original rhythm (one associated with it in an earlier familiarization phase) or in a new rhythm. Using this procedure, Jones et al. found that listeners had difficulty recognizing targets in the new rhythm, and tended to mistake the decoys that shared the target's pitch contour for targets, especially when they appeared in the target's original rhythm. Although these findings are consistent with the idea that contour and rhythm jointly influence memory for melody, they do not specifically address how this happens. In the present research, different melodies and rhythms were used to pursue this issue.
A construct that guided selection of melodies and rhythms in the present research is joint accent structure, which Jones (1987) proposed guides dynamic attending to auditory events in many different tasks (see, e.g., Boltz \& Jones, 1986; Jones, Boltz, \& Kidd, 1982; Martin, 1972; Monahan \& Carterette, 1985; Yeston, 1976). A joint accent structure is an accent/time hierarchy in which differentially weighted pitch and time accents are linked together by nested time relations (Jones, 1976). Relational properties of the joint accent structure determine, in part, expectancies about the pitch and time values of forthcoming events in a tune (Jones, 1976, 1981, 1982). Here we assume that: (1) pitch (e.g., melodic) and time (e.g., rhythmic) structures each contribute distinctive accents to this combined pattern, and co-occurring, namely coupled, accents receive more accent weight; (2) a melodic theme is specified primarily by time relationships among accented pitches of different weights; and (3) pitches that receive stronger accent weights reflect higher level properties of a joint accent structure, and because of their prominence in pitch or in time or both, they are more attentiongetting and hence more likely to establish the theme.

If people rely on such accent structures to selectively attend to a higher order melodic line, we can ask, "What changes in joint accent structure degrade listeners' ability to recognize a target melody?" and, relatedly, "What changes in joint accent structure continue to support thematic clarity?"

\section{The Experimental Rationale}

To address such questions, the experimental rationale involved manipulations of two aspects of joint accent structure in order to assess their impact on theme recognition. These aspects involved: (1) time relations among certain pitch accents; and (2) temporal phasing of pitch and time accents (accent coupling). Manipulations of these properties are illustrated with examples of recognition tunes in Figure 1.

Time relations among pitches in various target and decoy melodies were varied across the central segments of various recognition tunes with special attention to pitches marking contour changes. Essentially, we tampered with pitch placements in the middle of each tune by systematically varying the signed interval time values that obtained between onsets of central pitches and ones in the surrounding context. Consider, for example, the target melody depicted in Figures la and Id; its contour peaks are marked with key-related pitches denoted musically as $\mathrm{G}_{5}$, $\mathrm{G}_{\mathbf{4}}, \mathrm{A}_{\mathbf{4}}, \mathrm{C}_{\mathbf{4}}, \mathrm{F}_{\mathbf{4}}$, and $\mathrm{B}_{3}$. Such pitches are termed contourpitch accents, and they can differ in prominence or weight. ${ }^{2}$ The most prominent contour-pitch accent is $G_{5}$, which establishes its time relation to the opening tonic $\left(C_{4}\right)$ by virtue of its placement at the seventh serial location. Both the accent weight and the relative time value (e.g., with respect to the opening tonic) of such a pitch enter into hierarchical properties of this tune's joint accent structure and thus contribute to its thematic character. Similarities and differences among targets and decoys in the present research play on this idea. Thus, compare this tar- 

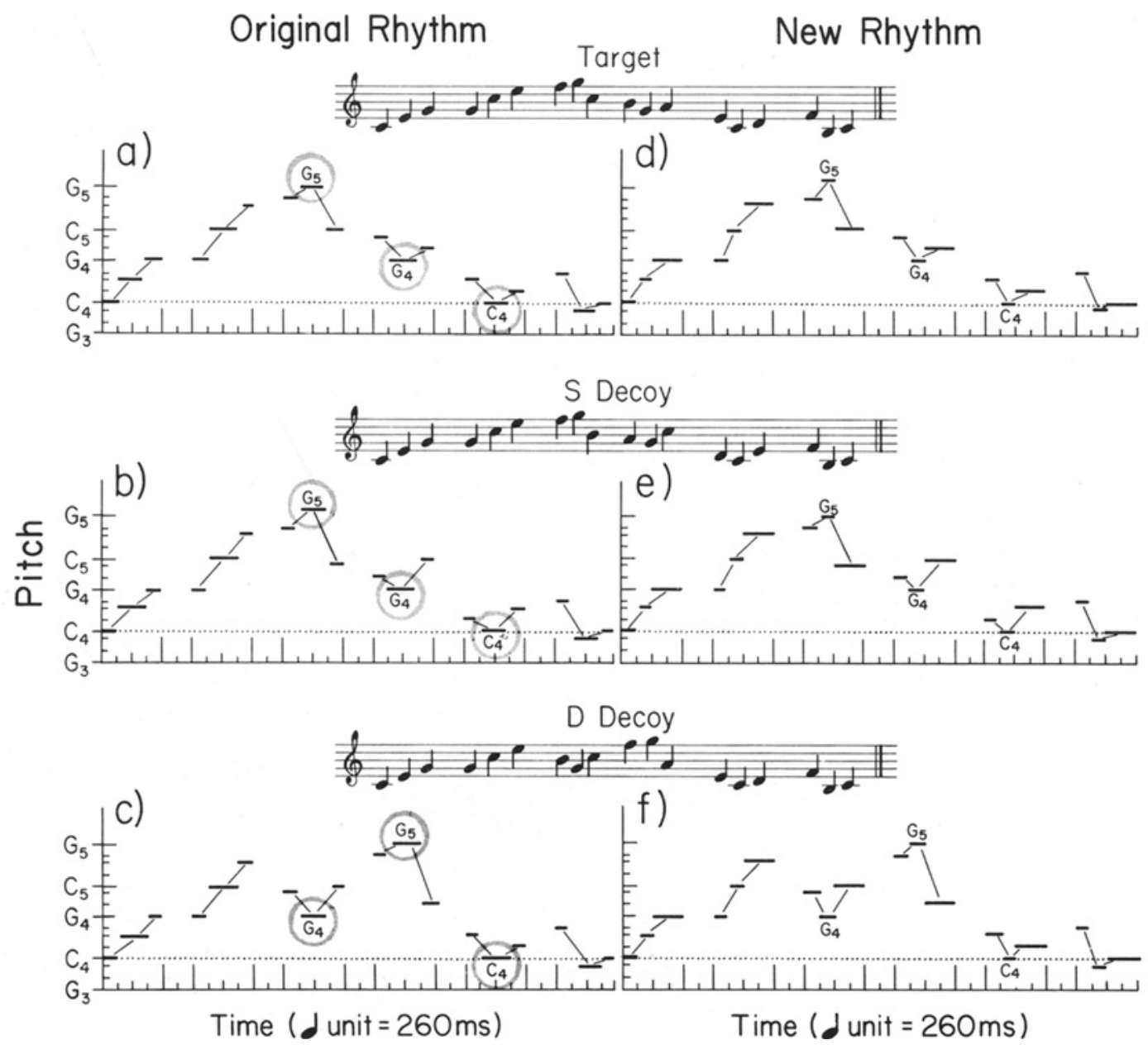

Figure 1. Target, same (S), and different (D) decoy melodies in both the original (a, b,c) and new rhythms (d, e, n). Circled notes are pitch-contour accents that are coupled with temporal accents (i.e., longer notes); these coupled accents occur in the original, but not in the new, rhythm (Experiment 1).

get to a decoy with the same pitch contour shown in Figures $1 \mathrm{~b}$ and $\mathrm{le}$. Although this decoy manifests numerous changes in the serial locations of pitches in its central portion, it nonetheless has the same contour as the target. In fact, most of the same contour-pitch accents occur at the same serial locations. This is most evident for the prominent contour-pitch accent, $\mathrm{G}_{5}$, which has the same time interval relation with the opening tonic in both tunes. By contrast, a decoy with a pitch contour that is different from the target's is shown in Figures 1c and 1f. It has more changes in the serial loci of its central pitches because some contour-pitch accents have also been shifted. Thus, the global pitch peak, marked by $\mathrm{G}_{5}$, is temporally shifted farther from the opening segment than it is in the other two tunes. ${ }^{3}$ In other respects, the two decoys share many features (see the Method section).

In sum, relatively long tonal melodies, which open and close with similar key-specific information (in this case $\mathrm{C}$ major) and which offer a higher order melodic line of contour-pitch accents, are systematically manipulated with respect to their relative time properties. The hypothe- sis of interest maintains that if listeners rely on time relations among these sorts of pitch accents to selectively track this theme, then same decoys should be mistaken for targets more often than different decoys.

To pursue this further, rhythm was manipulated so that in some cases it highlighted nonadjacent events associated with the higher order melodic line, whereas in other cases it did not. Given a joint accent structure analysis, it is possible to devise rhythmic contexts that add prominence to contour-pitch accents by correlating them with temporal accents; this was done with target melodies in the first phase of the experiments reported here. Each of three rhythmic figures was tailored to a target melody such that its time accents (e.g., lengthened durations) tended to coincide with the melody's contour-pitch accents. The rhythms were three simple and predictable durational patterns, each combining long (L) and short (S) durations with the $\mathrm{L}$ tone durations taken as temporally accented ones. They differed from one another only with respect to placement of the temporal accent (L) within successive three-tone groups, each group being identically punc- 
tuated by a pause (i.e., SSL pause SSL ..., SLS pause SLS ..., LSS pause LSS ...). Thus, whenever a time accent coincides with a pitch accent, accent coupling results (Jones \& Boltz, 1989). Coupled accents are assumed to have more attention-getting weight, particularly with untrained listeners (Monahan, Kendall, \& Carterette, 1987). In familiarization, all target melodies had several coupled accents by virtue of the rhythm assigned to them.

In recognition, rhythm was explicitly manipulated so that all tunes appeared both in their original (or a similar) rhythm, and so preserved accent coupling, and in a new (or dissimilar) rhythm where accent coupling did not occur. Figures $1 \mathrm{a}, \mathrm{lb}$, and $1 \mathrm{c}$ show accent coupling in targets, same decoys, and different decoys with the original rhythm (e.g., SLS); circled tones from the central segments of various melodies indicate weightier accents due to couplings with this rhythm. The same melodies appear in a new rhythm (SSL) in Figures 1d, le, and If. Note that temporal accents are phase-shifted relative to contour-pitch accents, resulting in a decoupling of these two kinds of accents. In the original rhythm, certain contour-pitch accents that participate in the target melody's theme are highlighted (coupled) whether or not they appear in the same serial location as in the target (same vs. different decoy). Similarly, in the new rhythm, pitch and time accents are decoupled in all melodies regardless of serial location of the relevant pitches.

Two contrasting hypotheses speak to rhythmic manipulations. One assumes that rhythm affects memory for melody simply by virtue of placement of pauses. Pauses segment each tune and can thus determine which lower order melodic groups are encoded as chunks (see, e.g., Deutsch, 1980; Dowling, 1973; Handel, 1984, 1989). However, in the present research, rhythm is manipulated in fairly constrained ways, and differential segmenting due to pause placements is precluded because the serial loci of pauses are identical for all rhythms. Thus, if melodic codes based on pause structure in familiarization do govern later melody recognition, a pause-segmentation hypothesis predicts that changing a melody's rhythm will have little systematic effect on recognition performance.

An alternative hypothesis, derived from a joint accent structure approach, holds that rhythmic highlighting boosts the accent weights of certain pitches and thus guides people's attending to the same thematic information both in familiarization and in recognition (Jones, 1987). If so, then rhythms that shift the relative locations of lengthened durations (temporal accents) within pause-based groups affect temporally selective attending because they lower accent weights. In the present context, if a new rhythm decouples pitch and time accents, it will decrease the likelihood of attending to theme-bearing, but nonadjacent, pitches. In contrast to the pause-segmentation account, this interpretation predicts that these rhythmic shifts will lower melodic discriminability.

Both the pause-segmentation hypothesis and the joint accent structure interpretation imply that melody perception depends on rhythm. However, evidence for interactions of melody with rhythm is mixed, with some report- ing significant interactions (e.g., Boltz \& Jones, 1986; Deutsch, 1980; Jones et al., 1987) and others not (e.g. . Palmer \& Krumhansl, 1987a, 1987b). Typically, multiple effects of rhythm on melodic structure have not been controlled, and general conclusions are therefore difficult. Because the present design represents an attempt to control some of these effects (e.g., pause placements), it sheds some light on this complex issue.

\section{EXPERIMENT 1}

Listeners were instructed to learn three different target melodies in Phase 1, a familiarization session. They were explicitly told to attend to the melody and ignore its rhythm. Furthermore, they were given no indication of a forthcoming recognition test of the tunes. In recognition (Phase 2), listeners were warned that melodies could appear in a new rhythm and were again advised to ignore rhythm and concentrate only on the melody. While the same rhythms were used in both familiarization and recognition phases, in recognition these durational patterns could be paired either with their original melodies and decoys (original rhythm) or re-paired with different melodies (new rhythm). In this way, a confounding factor of rhythmic novelty associated with rhythmic shifts in the new rhythm condition could be ruled out.

\section{Method}

\section{Subjects}

Twenty freshmen and sophomores from an introductory psychology course at Ohio State University participated in the experiment in return for course credit. All had 2-5 years of formal musical training. They were tested in groups of 1 to 4 .

\section{Design}

Two phases, a leaming or familiarization phase (Phase 1) and a recognition phase (Phase 2 ), were used. Only Phase 2 data were analyzed. Recognition accuracy was assessed using a $3 \times 2 \times 2$ mixed factorial design, for which three levels of melody (target, same, different) were crossed with two levels of rhythm (original, new). Counterbalance order (two leveis) was the only between-subjects variable. The primary dependent variable was a nonparametric signal detection measure of sensitivity, $A_{g}$. The $A_{g}$ metric required collapsing this design into two levels of melody (target vs. same and target vs. different), creating $2 \times 2 \times 2$ mixed factorial design for $A_{8}$ and its related bias measure $(B)$.

\section{Stimulus Materials}

Nine melodies formed three melodic sets, with each set based on a target and two decoys (same, different). All melodies were constructed of 18 square-wave tones of equivalent intensities. They were combined with different rhythms. Below, we outline melodic (pitch) sequences (targets, decoys); rhythms; and pairings of melodies with rhythms. The melodies in Sets 1, 2, and 3 are listed in the Appendix.

Melodies: Targets. Target melodies were constructed in two musical keys: $\mathrm{F}$ major (familiarization) and $\mathrm{C}$ major (recognition). To reinforce these tonalities, each target melody began and ended on its keynote pitch (i.e., either $F$ or $C$ ), opened with prominent tonal relations (e.g., involving notes of the tonic triad), and closed with the final tonic resolving through a leading tone (i.e., $E$ and $B$, respectively, in the keys of $F$ and $C$; see Butler \& Brown, 1984). The three target melodies differed from one another in terms of both 
interval and contour-pitch relationships. Respectively, they contained four, six, and eight contour changes, plus distinctive opening segments. Figures la and 1d illustrate one of these; others appear in the Appendix.

Melodies: Decoys. For each target melody in a set of three melodies, two decoys were constructed in the key of $\mathrm{C}$ major for the recognition phase. These were: a same contour decoy and a different contour decoy. In each melody set, same and different decoys always shared the following properties with their target: (1) the same tones, with the serial locations of some changed; over the three melody sets, the number of location changes of pitches that were not at coupled accent locations were equated for same and different decoys (a total of 10 changes each), although within each melody set, same and different decoys differed, respectively: five and two changes (Set 1); two and four changes (Set 2); three and four changes (Set 3 ); (2) the same number of contour changes; (3) the same initial six and final three tones and their orderings (to establish the same key); (4) similar, but not identical, pitch intervals: the averages of median pitch intervals were 3,3 , and 2.66 ST for targets, same, and different decoys, respectively; (5) a majority of the same pitches marking contour accents (i.e., theme-bearing pitches); and (6) identical coupled (i.e., contour-pitch plus time) accents in target, same, and different melodies: $\mathrm{G}_{\mathbf{4}}, \mathrm{C}_{\mathbf{4}}$, and $\mathrm{G}_{5}$ (Set 1 ); $G_{3}, C_{4}$, and $C_{5}($ Set 2$) ; C_{4}$ and $E_{5}$ (Set 3 ).

Decoys differed from their respective targets with regard to when central pitches of various weights occurred relative to the melody's opening notes. With the opening tonic (Serial Location 1) as a temporal referent, time intervals associated with particular pitches at coupled accent points are identical for targets and same decoys but not for different decoys. Constraints on target and decoy construction resulted in same and different decoys differing in the total number of serial location changes: a total of 11 for same decoys and 16 for different decoys.

Rhythms. Three different recurrent durational patterns were used. All consisted of four durations, where each interval could be short (S) or long (L). Each rhythm divided the 18-tone pitch sequence into successive three-tone phrases because the fourth time interval was always a long pause. The other three intervals determined tone
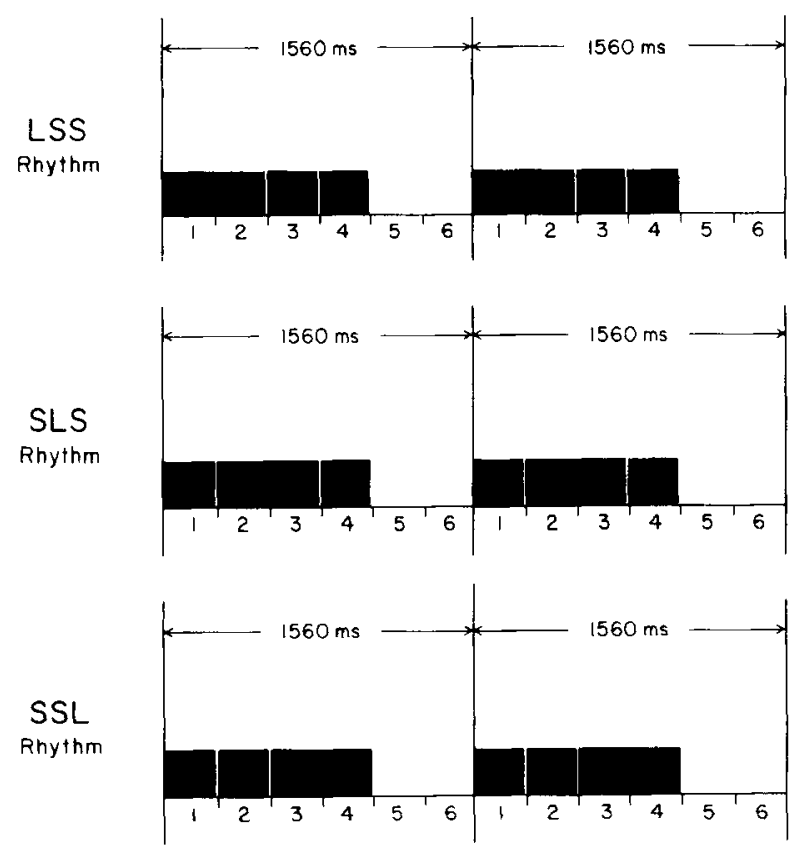

Figure 2. Three different durational patterns used in Experiment 1: LSS, SLS, and SSL. All contain six SOAs of $260 \mathrm{msec}$ (S) per higher order period (1,560 msec) and a pause duration (rest) of $520 \mathrm{msec}(L)$. durations as shown in Figure 2: SSL, SLS, and LSS. The long duration refers to an SOA $(520 \mathrm{msec})$ that was always twice the length of the shorter duration SOA $(260 \mathrm{msec})$. All SOAs included a 10 -msec off time between adjacent tones. The pause or musical rest between recurrences of each rhythm was always $520 \mathrm{msec}$, yielding a constant higher order time period of $1,560 \mathrm{msec}$ for each threenote phrase (plus pause); this structure was used for all three thythms.

Melody -rhythm pairings. In Phase 1, each rhythm was paired with a different target melody following preliminary assumptions about accents and tonality. Pairings also relied on judgments of an independent group of four expert listeners asked to rate the tunes with respect to accents (contour and temporal) and tonality (key). Phase 1 pairings were designed to maximize the correlation of contour-pitch accents with temporal accents; they contained two or three coupled time and pitch accents within the distinctive central core of nine tones (no more than five throughout). All were judged to be in the same key.

In Phase 2, each target melody appeared in two thythms: the original rhythm and a new rhythm. In the original rhythm, each target melody and its respective decoys were paired with the same rhythm that accompanied targets during familiarization; in the new rhythm condition, the three Phase 1 rhythms were re-paired with different target melodies and their corresponding decoys in order to phase shift pitch and time accents. Additional constraints of re-pairing for the new rhythm were: (1) A new melody-rhythm combination should not change the sense of key (preliminary ratings of experts guided these selections); (2) the new rhythm should reflect a change of two of the four durations within a group; and (3) duration and locus of the pause within the melodic string remained unchanged. Thus, the new rhythm mappings were: SSL pause $\rightarrow$ LSS pause; SLS pause $\rightarrow$ SSL pause; LSS pause $\rightarrow$ SLS pause.

\section{Procedure}

The entire experiment consisted of two phases: a familiarization phase (Phase 1) and a recognition phase (Phase 2).

Phase 1: Familiarization. Recorded instructions stressed a distinction between melody (as a pitch sequence) and rhythm (as a durational pattern). Listeners were urged to ignore the rhythm and differentiate the three target instances on the basis of the melodies only. They were not informed about the recognition phase.

Each Phase 1 trial was initiated by a warning tone $(3250 \mathrm{~Hz})$, followed after $2 \mathrm{sec}$ by a target melody and then a 5-sec response interval. Over the 27 trials, each target appeared nine times. On each trial, the listener had to identify the target melody (using one of three letters: $\mathrm{A}, \mathrm{B}$, or $\mathrm{C}$ ) and give a confidence rating of his or her choice $(1=$ most confident; $7=$ least confident).

Melodies appeared with different pitch and time transpositions (applied uniformly to the whole sequence). These ensured that listeners would respond to melodic and rhythmic invariants that differentiated the targets. Such transformations commonly accompany recurrences of a given theme in music: a pitch shift up or down one octave; and a ratio time transformation that sped or slowed the melody by $2 / 3$ or $4 / 3$, respectively. Targets were subjected to both, one, or none of these transformations. Over the 27 trials, melodies were presented randomly, in one of two counterbalance orders, with the constraint that no more than two instances of a given target melody occur consecutively.

The Phase 1 session ended with three "hum" trials, during which the subjects covertly hummed each target after its untransformed presentation and then rated their confidence in the accuracy of their reproduction. If anyone in a group indicated low confidence, this procedure was repeated. A 15 -min break followed, during which the subjects performed an unrelated task.

Phase 2: Recognition. In recognition, the subjects were told via recorded instructions that they would hear the earlier melodies plus new ones. The instructions cautioned that they attend only to the pitch sequence and ignore rhythm, because sometimes melodies would occur in a new rhythm. 
In this session, each trial was signaled by a 1-sec, high-pitched warning tone, followed after 2 sec by a pattern. The listener had $5 \mathrm{sec}$ to indicate whether the melody was "old" (i.e., a target) or "new" (i.e., any decoy) and to register a confidence rating on the same 7-point rating scale as before.

All melodies occurred in the key of $C$ major; none was trans formed in pitch or tempo. In a total of 48 trials, the subjects received 24 targets and 24 decoys equally often in both rhythms. Four repetitions of each of the three target melodies appeared in each of the two rhythmic conditions (i.e., 12 targets per rhythmic condition). The same and different decoys each accounted for 6 trials in each rhythmic condition (two repetitions per melodic instance). Melodies were presented randomly, with the constraint that no melodic or temporal instance recur over more than 2 consecutive trials.

Scoring. Recognition was analyzed in terms of $A_{g}$ and bias $(B)$ scores. The $A_{g}$ score involves a nonparametric ROC score based on computation of the trapezoidal rule (Bamber, 1975; Pollack, Norman, \& Galanter, 1964) adapted by Davison and Jagacinski (1977). The $A_{g}$ score estimates unbiased recognition accuracy (sensitivity) in the two-choice case, for which an $A_{g}$ value of 1.00 reflects perfect discrimination and a value of 50 reflects random guessing. The bias measure roughly estimates the median confidence level score when the 7-point scale is converted to a 15-point scale ranging from 1 (sure-old) to 14 (sure-new); a score of 7.00 represents no bias. Both $A_{g}$ and $B$ scores were generated by randomly dividing the target trials into halves, and comparing a subject's response and confidence rating on the targets to the same and different decoys across each rhythmic condition. This analysis essentially collapses the trilevel melody factor into two levels, subsequently referred to as target:same and target:different. Supporting analyses involve accuracy scores, namely hit rates (proportion correct to targets) and correct rejections (proportion correct to decoys; note that the false alarm rate is simply 1 - the proportion correct to decoys).

\section{Results}

For all dependent measures, analyses involving the presentation order of stimuli showed this counterbalance variable to be nonsignificant. Thus all of the presented results have been collapsed across this factor.

\section{Sensitivity/Accuracy}

Variations in melodic structure systematically affected discriminability. The subjects more often confused same decoys with targets than they did different decoys. Table 1 presents mean $A_{g}$ values as a function of melodic condition (target:same and target:different) as well as rhythm (original vs. new). The $A_{g}$ metric is based on comparing performance of targets (signals) with a set of decoys (noise). When the set involved same decoys as noise, the mean $A_{g}$ was .719 , whereas when different decoys supplied noise, the mean $A_{g}$ was $.835[F(1,18)=16.25$, $\left.M S_{\mathrm{e}}=16.620, p<.001\right]$.

Table 1

Mean $A_{1}$ and Bias Scores for Experiment 1

\begin{tabular}{|c|c|c|c|c|c|c|}
\hline \multirow[b]{3}{*}{ Rhythm } & \multicolumn{4}{|c|}{ Melody } & & \\
\hline & \multicolumn{2}{|c|}{$\begin{array}{l}\text { Target vs. } \\
\text { Same Decoy }\end{array}$} & \multicolumn{2}{|c|}{$\begin{array}{c}\text { Target vs. } \\
\text { Different Decoy }\end{array}$} & \multicolumn{2}{|c|}{ Means } \\
\hline & $A_{8}$ & Bias & $A_{g}$ & Bias & $A_{g}$ & Bias \\
\hline Original & .823 & 5.60 & 877 & 5.80 & .850 & 5.70 \\
\hline New & .615 & 7.75 & .794 & 9.15 & .704 & 8.45 \\
\hline Means & .719 & 6.68 & .835 & 7.48 & & \\
\hline
\end{tabular}

Table 2

Mean Proportion Correct for Experiment 1 Melody

\begin{tabular}{lcccc} 
& \multicolumn{3}{c}{ Melody } & \\
\cline { 2 - 4 } Rhythm & Target & $\begin{array}{c}\text { Same } \\
\text { Decoy }\end{array}$ & $\begin{array}{c}\text { Different } \\
\text { Decoy }\end{array}$ & Means \\
\hline Original & .864 & .680 & .798 & .781 \\
New & .584 & .680 & .890 & .718 \\
Means & .724 & .680 & .844 & \\
\hline
\end{tabular}

Rhythmic shifts also affected performance. Although pause segmentation is the same for original and new rhythms, subjects were better at discriminating melodies in the former. As measured by $A_{g}$, recognition was significantly better in the original rhythm than in the new rhythm [mean $A_{\mathrm{g}}=.850$ vs. $.704 ; F(1,18)=14.43, M S_{\mathrm{e}}=$ 29.556, $p<.0025]$.

Melody recognition is also differentially affected by rhythm, as is evidenced by a significant interaction of melody with rhythm $\left[F(1,18)=4.48, M S_{\mathrm{e}}=17.77\right.$, $p<.05]$. Disproportionately poor performance occurred when the subjects had to discriminate targets from same decoys in a new rhythm (mean $A_{g}=.615$ vs. .794, .823 , and .877 in the other conditions). Poor discriminability can come from either low target recognition accuracy or from errors to same decoys or both. The accuracy data (Table 2) suggest that the culprit here is poor target identification levels in the new rhythm. Error rates to same decoys (i.e., 1 - proportion correct; namely, false alarms) are identical in the two rhythms. Taken together, these findings tell us two things: (1) The new rhythm renders a melodic theme less attention-getting and thus increases identification errors in the target melody; and (2) the original thythm makes discrimination of targets from same and from different decoys roughly similar, a fact that argues against the possibility that different decoys are inherently more discriminable from targets than same decoys because they contain distinctive interval differences.

An interaction of melody with rhythm in proportion correct scores is also significant $\left[F(2,36)=25.30, M S_{\mathrm{e}}=\right.$ $.0148, p<.0001\}$. Although accuracy is lower with same decoys than with different decoys in both rhythms, post hoc analyses (Tukey's HSD) show that these differences were significant only in the new rhythm. We will return to this point.

\section{Bias}

A bias score of 7 reflects unbiased judgments, while $B$ scores larger than 7 indicate bias for saying "new." Table 1 shows mean $B$ scores as a function of the melody and rhythm conditions.

Rhythm biases judgments. People tend to judge any melody (target or decoy) to be familiar (i.e., "old" with mean $B=5.70$ ) when it appears in the original rhythm. Similarly if it appears in the new rhythm, they are more likely to judge a melody "new' [mean $B=8.45 ; F(1,18)=$ $21.75, M S_{\mathrm{c}}=6.96, p<.0005 \mathrm{]}$. This finding is not surprising. It suggests that changes in accuracy arise partly from the biasing influence of rhythm. 


\section{Discussion}

Clearly, both melodic and rhythmic structure affect delayed melody recognition. Listeners' memories of relatively long, rhythmically patterned tunes are systematically influenced by the melodic variations associated with the two kinds of decoys used, thus substantiating the influence of contour timing information on melodic discrimination. Furthermore, even when warned to ignore rhythm, listeners cannot do this. Shifts in rhythm significantly lower listeners' abilities to distinguish targets from decoys, despite the fact that pause structure is held constant. Although these findings are consistent with a joint accent structure analysis, they do not conclusively establish that accent coupling, which is preserved only by the original rhythm, is responsible for these effects. This issue is pursued in Experiment 2.

The finding that average listeners are more often fooled by same than by different decoys extends previous findings regarding effects of contour, which were based on shorter melodies and briefer retention periods (e.g., Dowling \& Fujitani, 1971; Monahan et al., 1987). Here, contour changes are reinforced by tonally significant pitches and thus effectively instantiate a memorable theme, which, for these listeners, lasts over a substantial retention interval.

It is interesting that differences among same and different decoys are more marked in the new rhythm than in the original one. In fact, in the original rhythm, same and different decoys are roughly equivalent in discriminability, which requires an explanation. Because thematically relevant pitches are presumably weighted more in the original rhythm, one might expect that listeners would take same decoys to be targets more often in this condition. In fact, such a finding did obtain in Jones et al. (1987), where listeners made disproportionately more false alarms to same than to different decoys in the original rhythm. Why didn't preserving the original rhythm boost error rates to same decoys in the present case? One explanation involves the fact that in the present study, rhythms with accent coupling were used, whereas in the Jones et al. experiments, they were not. It is possible that this highlighting serves more to facilitate discrimination of differences between a decoy and its target than to enhance their similarities. Another explanation also concerns the rhythms used. Jones et al. employed rhythms in which durational patterns were more heterogeneous as a set than those in the present research. Thus, re-pairing of rhythms in that study could have heightened the influence of any single distinctive rhythm on melody perception. If so, listeners in the Jones et al. study might have been more confused by same decoys when they appeared in the target's original rhythm as opposed to a very dissimilar one. We pursued the idea of rhythmic similarity in Experiment 2.

\section{EXPERIMENT 2}

In Experiment 2, we relied on the same design and strategy as in Experiment 1 to pursue two questions about the effect of rhythmic highlighting on long-term memory for melodies. The first question concerns effects of rhythmic similarity when listeners confront melodies in novel rhythms. The second explores the degree to which rhythmic similarity effects, if present, depend on the listener's familiarity with the target melodies in their original rhythms.

The term rhythmic similarity refers to the extent to which a new rhythm shares relevant temporal properties of the melody's original rhythm. For example, if accent coupling contributes to the superiority of the original rhythm in Experiment 1, then similar rhythms that preserve such aspects of familiar joint accent structure should also enhance melodic discriminability in Experiment 2.

We explore this by manipulating rhythmic similarity in terms of the way a rhythm fits with a melody as suggested by a joint accent structure analysis. Similarity depends here on the number and the kind of tone durations changed by a rhythmic shift (from Phase 1 to Phase 2). A new set of Phase 2 rhythms was used to realize these criteria in Experiment 2. Although distinctly different from the Phase 1 rhythms, when paired with Phase 1 melodies, these rhythms provided different degrees of similarity. Table 3 shows two different similarity mappings of Phase 1 and Phase 2 rhythms: similar and dissimilar rhythmic shifts. A similar rhythm involves changing two time intervals per pause-defined group, including the pause; a dissimilar rhythm involves changing all intervals in each pause-defined group. For example, the shift of LSS long pause ... to LLS short pause ... is a similar rhythm shift, whereas changing it to SLL short pause ... is a dissimilar rhythm shift. In addition, similar rhythm conditions do not phase shift pitch and time accents, whereas dissimilar rhythm conditions do. Consequently, similar rhythm conditions preserve accent coupling and dissimilar rhythm conditions do not (see Table 3). In this way, rhythmic similarity is not defined independently of what a rhythm does to a melody and its pitch accents; rather, it is assessed in terms of commonalities of pitch/time accent patterns. These constraints permit relevant comparisons of performance in the similar and dissimilar rhythm conditions to that in the original and new rhythm conditions, respectively, of Experiment 1.

The similarity manipulation captures certain composing techniques whereby listeners are made to experience

Table 3

Rhythmic Shift Mappings for Experiment 2

\begin{tabular}{|c|c|c|}
\hline \multicolumn{3}{|c|}{ Similar Rhythm Condition } \\
\hline Phase 1 & & Phase 2 \\
\hline S S L* & 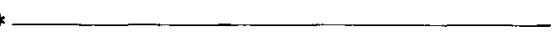 & $L^{*} \mathbf{S} \quad L^{s}$ \\
\hline $\mathbf{S} \quad L^{*} \mathbf{S}$ & & S $L^{*} L^{s}$ \\
\hline$L^{*} S \mathrm{~S}$ & & $\mathrm{~L}^{*} \mathrm{~L}^{\mathrm{s}} \mathrm{S}$ \\
\hline & Dissimilar Rhythm Condition & \\
\hline Phase 1 & & Phase 2 \\
\hline $\mathrm{S} S \mathrm{~L}^{*}$ & - & $\mathrm{L}^{*} \mathrm{~L}^{\mathrm{s}} \mathrm{S}$ \\
\hline $\mathbf{S} \mathbf{L} * \mathbf{S}$ & - & $\mathrm{L}^{*} \mathrm{~S} \quad \mathbf{L}^{\mathrm{s}}$ \\
\hline$L^{*} S \quad S$ & & $S \quad L^{*} L^{s}$ \\
\hline
\end{tabular}

*Primary temporal accent. 'Secondary temporal accent. 
different degrees of thematic reminiscence, depending on the rhythm in which some melody or its variation recurs within a larger composition. We anticipate, therefore, that both kinds of rhythmic shifts in Experiment 2 will lower a listener's discrimination of target melodies relative to its original rhythm. However, the similar rhythm shifts preserve higher order aspects of a tune's joint accent structure such as its accent coupling properties, and since these are assumed to be more prominent in guiding attending and remembering, the similar rhythm condition should yield better target recognition than the dissimilar rhythm condition, much as the original rhythm did in Experiment 1 . Thus, the similarity manipulations allow us to consider what changes in a joint accent structure tend to preserve target recognition and what ones do not.

Relatedly, we can also determine whether the general context provided by new rhythms affects listeners' responses to thematic variations instantiated by same decoys. Data from Experiment 1 suggest that same decoys are not significantly more confusing than different decoys when both appear in the target's original rhythm. However, novel rhythms may encourage listeners to focus largely on higher order properties of the joint accent structure; if so, same decoys may be more often mistaken for targets in the similar rhythm conditions than in dissimilar conditions. While both similar and dissimilar rhythm conditions introduce joint accent structure changes, the former preserves important higher level relations involving coupled accents.

Finally, in Experiment 2, we also looked at perceptual learning. Listeners' familiarity with targets in their original rhythm was systematically varied across two separate studies (Experiments 2A and 2B). Common sense led to the expectation that performance should be better with more Phase 1 training. However, we were also interested in discovering whether differential effects of rhythm or melody variables would appear. That is, do people immediately respond to certain properties of a joint accent structure associated with more prominent accents (i.e., coupled accents)? If so, they should display disproportionately high performance in similar rhythm conditions even with relatively unfamiliar tunes.

\section{Method}

The method of Experiment 2 was identical to that of Experiment 1 , with the following exceptions:

\section{Subjects}

Ohio State University freshmen and sophomores were assigned randomly to two counterbalance orders in each of two experiments. In Experiment $2 \mathrm{~A}, 16$ subjects served in each order $(N=32)$; in Experiment $2 \mathrm{~B}, 13$ subjects served in each order $(N=26)$.

\section{Design}

The design for each experiment was identical to that of Experiment 1 , provided that the rhythm variable is taken to be the rhythmic similarity manipulation.

Experiment 2A: High familiarity. This study was identical to Experiment 1, with the exception that the 48 Phase 2 trials involved new rhythms based on combinations of two $L$ durations and one $S$ tone duration, plus a pause of $S$ duration. Phase 1 consisted of 27 trials

Experiment 2B: Low familiarity. This study was identical to Experiment 2A, with the exception that fewer familiarization trials were used. In Phase 1, participants heard only one presentation of each target melody in its rhythm and then received the "hum" trials. They received no melody-identification training trials. This study was conducted approximately 6 months after Experiment $2 \mathrm{~A}$ had been completed.

\section{Stimulus Materials}

Phase 2 rhythms are presented in Table 3. The SOAs for the two $L$ durations remained $520 \mathrm{msec}$ with $10 \mathrm{msec}$ off time; the $S$ tone duration and pause were $260 \mathrm{msec}$. Loci of temporal accents were determined both by conventional analyses of primary and secondary accents and on the basis of judges' ratings of these rhythms, both in monotone and target melody contexts. Although ratings were somewhat variable, the locations selected generally qualified as either primary or secondary temporal accents.

The mapping rules used to define similar and dissimilar rhythm conditions, given Phase 1 rhythms, are shown in Table 3. They observed the following criteria: similar rhythm conditions-changed two durations per recurrent thythmic group (one tone and the pause), while preserving identical serial locations of temporal accents in both Phase 1 and Phase 2 rhythms. In two of the three similar rhythms, this involved primary accents; in the third, a secondary accent was involved (a mapping involving two durational changes, which always preserves the temporal accent, was not possible with this rhythmic set); dissimilar rhythm conditions-over all four durations (per group), reverse $L$ duration and $S$ durations. A by-product of this was that the resulting Phase 2 rhythms always phase shifted the serial location of the primary temporal accent of the Phase 1 rhythm by a time constant. As in Experiment 1, both mappings preserved serial locations of pauses (musical rests) within melodies.

\section{Results}

The results of Experiments $2 \mathrm{~A}$ and 2B, collapsed over counterbalance order (a nonsignificant variable), are presented in Tables 4 and 5 . Initially, these data will be discussed separately, because they arise from experiments conducted 6 months apart. Subsequently, the performance with familiar tunes (2A) will be compared with the performance with unfamiliar ones (2B).

Table 4

Mean $A_{\varepsilon}$ and Bias Scores as a Function of Target Familiarity in Experiment 2

\begin{tabular}{|c|c|c|c|c|c|c|}
\hline \multirow[b]{3}{*}{ Rhythm } & \multicolumn{4}{|c|}{ Melody } & \multirow{2}{*}{\multicolumn{2}{|c|}{ Means }} \\
\hline & \multicolumn{2}{|c|}{$\begin{array}{l}\text { Target vs. } \\
\text { Same Decoy }\end{array}$} & \multicolumn{2}{|c|}{$\begin{array}{c}\text { Target vs. } \\
\text { Different Decoy }\end{array}$} & & \\
\hline & $A_{g}$ & Bias & $A_{8}$ & Bias & $A_{g}$ & Bias \\
\hline \multicolumn{7}{|c|}{ High Familiarity: Experiment $2 \mathrm{~A}$} \\
\hline Similar & .713 & 5.56 & .834 & 6.66 & .773 & 6.11 \\
\hline Dissimilar & .649 & 7.19 & .778 & 8.16 & .713 & 7.67 \\
\hline Means & .681 & 6.37 & .806 & 7.41 & & \\
\hline \multicolumn{7}{|c|}{ Low Familiarity: Experiment 2B } \\
\hline Similar & .714 & 6.04 & .733 & 7.27 & .724 & \\
\hline Dissimilar & .665 & 5.58 & .756 & 9.31 & .710 & $\begin{array}{l}0.03 \\
7.44\end{array}$ \\
\hline Means & .689 & 5.81 & .744 & 8.29 & & \\
\hline
\end{tabular}


Table 5

Mean Proportion Correct as a Function of Target Familiarity in Experiment 2

\begin{tabular}{lcccc}
\hline & \multicolumn{4}{c}{ Melody } \\
\cline { 2 - 3 } Rhythm & Target & $\begin{array}{c}\text { Same } \\
\text { Decoy }\end{array}$ & $\begin{array}{c}\text { Different } \\
\text { Decoy }\end{array}$ & Means \\
\hline \multicolumn{5}{c}{ High Familiarity: Experiment } \\
Similar & .810 & .594 & .786 & .730 \\
Dissimilar & .627 & .667 & .818 & .704 \\
Means & .718 & .630 & .802 & \\
& Low Familiarity: Experiment $2 \mathrm{~B}$ & \\
Similar & .686 & .587 & .702 & .658 \\
Dissimilar & .606 & .535 & .830 & .657 \\
Means & .646 & .561 & .766 & \\
\hline
\end{tabular}

\section{Experiment 2A: High Familiarity}

The recognition performance of listeners with substantial familiarity with the target tunes is summarized in Tables 4 (mean $A_{g}$ and $B$ ) and 5 (mean proportion correct).

Sensitivity/accuracy. Relative to Experiment 1, novel rhythms did reduce the listeners' abilities to discriminate melodies somewhat. However, melodic structure had comparable effects in both experiments. Listeners were poorer at distinguishing targets from same decoys than from different decoys $\left[A_{g}\right.$ values of .681 vs. $.806 ; F(1,30)=$ $\left.16.86, M S_{\mathrm{e}}=29.787, p<.0005\right]$. Rhythmic similarity also had a significant effect on performance; shifting melodies to a similar rhythm produced better discriminability than shifting them to a dissimilar one (see Table 4) $\left[F(1,30)=14.38, M S_{\mathrm{e}}=7.879, p<.001\right]$. Nevertheless, in both rhythms, the listeners were significantly above chance levels in distinguishing targets from decoys (i.e., chance $A_{g}$ is .50).

Perhaps the most important finding is that the interaction of rhythm with melody observed for discriminability scores in Experiment 1 disappeared in this study. A comparison of Tables 1 and 4 explains why. Although the listeners were best with similar rhythms, relative to Experiment 1 levels they were somewhat poorer in distinguishing targets from same decoys in rhythms with accent coupling (i.e., similar vs. original rhythms). This lack of interaction in $A_{g}$ is more in line with the results of Jones et al. (1987).

Failure to find the interaction of $A_{g}$ scores as in Experiment 1 derives from two sources, as substantiated by accuracy scores in Table 5. First, as anticipated, when same decoys appeared in novel, but similar, rhythms, they indeed fooled people more than in novel, but dissimilar, rhythms. As predicted, error rates to decoys in this study were highest to same decoys in the similar rhythm condition. The same decoys were significantly more confusing than different decoys not only in the dissimilar rhythm condition but, in contrast to Experiment 1, also in the similar thythm condition (Tukey $H S D=.156$, $p<.01)$. The second contributing factor was mentioned above: Target identification accuracy in similar rhythm conditions was slightly poorer than in the original rhythm of Experiment 1. Thus, while discriminability scores do not show an interaction of melody with rhythm, accuracy scores do $\left[F(2,60)=9.92, M S_{\mathrm{c}}=.0313, p<.0005\right]$. Contributing to this effect is the fact that although correct identifications of same decoys rose in the dissimilar rhythm condition, target identification accuracy dropped in these rhythms.

Finally, accuracy measures reflect a combination of biasing influences and discriminability. In Experiment 2A, some of the changes in accuracy levels as a function of rhythm arose from its biasing potential.

Bias. Table 4 shows mean $B$ scores as a function of changes in melody and rhythm. The bias findings replicate those of Experiment 1, in that rhythm has the primary influence on performance. Melodies appearing in similar rhythms tend to evoke "old" responses, and those in dissimilar rhythms to evoke "new" responses $[F(1,30)=$ 9.56, $\left.M S_{c}=8.18, p<.005\right]$. The most unbiased performance levels involved the set of targets and different decoys in the similar rhythm condition and the set of targets and same decoys in the dissimilar rhythm condition.

\section{Experiment 2B: Low Familiarity}

The performance of listeners who had less familiarity with target tunes also is summarized in Tables 4 (mean $A_{g}$ and $B$ scores) and 5 (mean proportion correct scores).

Sensitivity/accuracy. The pattern of $A_{g}$ scores with less familiar tunes bears some resemblance to that found with familiar ones, but the effects of melody and rhythm are more modest with no statistically significant impact (Table 4). Two aspects of these null findings deserve comment: (1) Listeners are not disproportionately better in the similar rhythm conditions; and (2) they are relatively poor overall in differentiating different decoys from targets.

Supplementing these findings, the accuracy scores indicate that the listeners did, in fact, err more often with same than with different decoys in both rhythms $[F(2,48)=$ $\left.11.47, M S_{\mathrm{e}}=.048, p<.0001\right]$. However, rhythm continued to have no overall effects on accuracy (the mean proportions correct for similar and dissimilar rhythm conditions were roughly equivalent). With less familiar tunes, similar rhythms actually produce an improvement in listeners' chances of spotting same decoys relative to dissimilar rhythms; accuracy levels to same decoys were near chance in the dissimilar rhythm condition. However, similar rhythm conditions also decreased the listeners' chances of spotting different decoys as such relative to the dissimilar rhythm conditions. A significant interaction of rhythm with melody resulted $[F(2,48)=$ $\left.7.72, M S_{e}=.022, p<.002\right]$. These error rates appear to reflect a combination of biasing influences of contourpitch accent structure and rhythm.

Bias. In contrast to listeners familiar with targets, these listeners were biased by differences in melodic structure $\left[F(1,24)=25.26, M S_{\mathrm{e}}=6.33, p<.0001\right]$. The same decoys evoked more "old" judgments than did the different decoys. The mean bias for the target:same set was $B=5.81$, whereas that for the target:different set was much higher $(B=8.29)$. Also in contrast with Experi- 
ment $2 \mathrm{~A}$, rhythm only modulated these effects, making them most evident in the dissimilar rhythm conditions; it had no overall influence. This interaction of melody and rhythm is significant $\left[F(1,24)=5.12, M S_{\mathrm{e}}=7.94, p<\right.$ $.05]$. Post hoc comparisons indicated that the difference within the dissimilar rhythm condition was significant (Tukey HSD $=2.09, p<.05$ ). Thus, the bias measures fleshed out effects of rhythm and melody on accuracy scores that were considered earlier.

\section{Experiment 2A versus 2B: Familiarity Effects}

Listeners who were more familiar with target tunes were not substantially better overall in differentiating them from decoys in recognition. The main effect for the familiarity effect on $A_{g}$ scores was nonsignificant ( $F=1.18$, $p<.28)$. Furthermore, as a variable in the combined $A_{g}$ analysis, familiarity did not significantly qualify the effects of either rhythm or melody. Not surprisingly, although both melody and rhythm had significant effects in this combined analysis $\left[F(1,54)=16.89, M S_{\mathrm{e}}=\right.$ $27.614, p<.0001$, for melody; $F(1,54)=7.25, M S_{e}=$ $10.443, p<.01$ for rhythm], neither interacted with familiarity level (Experiment $2 \mathrm{~A}$ vs. $2 \mathrm{~B}$ ). In fact, the listeners with different degrees of target familiarity were virtually identical in their abilities to distinguish same decoys from targets in similar rhythms (mean $A_{g}$ scores of .714 vs. .713). This finding, together with the fact that a combined analysis yielded no significant interaction of rhythm or melody with familiarity, suggests that some immediate advantages accrue to preserving rhythmic and pitch-contour similarities. Nevertheless, on the average, these early differences are slight compared with those observed with familiar tunes.

Where do these two groups of listeners differ, if they do? Overall accuracy improves significantly with familiarity (from proportion correct of .658 to .717) $[F(1,54)=$ $\left.5.77, M S_{\mathrm{c}}=.0528, p<.02\right]$. The most striking change arises from improvements in target recognition in similar rhythm conditions, and this contributes to a significant three-way interaction of familiarity level with melody and rhythm $\left[F(2,108)=4.56, M S_{\mathrm{e}}=.027, p<.025\right]$. The accuracy and bias scores in both combined and separate analyses suggest that listeners who are less familiar with target melodies are more biased overall by melodic differences among to-be-recognized tunes and that they come to rely more heavily on rhythm with familiarity. Although large initial effects of rhythm were not evident, rhythm did exert some early influences on the listeners' sensitivity to pitch contour. Furthermore, these effects seemed to intensify with the listeners' experience. This was evident in the listeners' relative performance on targets and same decoys as a function of familiarity: Error rates declined with targets in the similar rhythm condition, whereas they did not with same decoys. In fact, improvement with same decoys as a function of familiarity occurred largely with the dissimilar rhythm conditions, in which proportions correct climbed to .667 from .535 . This pattern of findings suggests that there is an immedi- ate sensitivity to pitch contour information that can persist if it is rhythmically reinforced.

\section{Discussion}

People learn to extract from relatively long melodies enough information about their pitch structure to recognize them in novel rhythms, especially if these rhythms share certain temporal properties with their original rhythms. This experiment sheds light on three aspects of this phenomenon. Two concern the way rhythmic similarity affects recognition of targets and decoys, respectively. The third is related to listeners' differential use of rhythm with perceptual learning (familiarity).

People recognize targets best when these tunes reappear in similar rhythms. While they are less adept with novel rhythms than when such tunes appear in their original rhythm, people nonetheless correctly recognize targets around $80 \%$ of the time (relative to $86 \%$ in the original rhythm). Thus, certain rhythmic shifts can support relatively high levels of theme recognition. Undoubtedly, a key element in this effect is the novel rhythm's accent coupling potential.

Design constraints in Experiment 2 meant that the similar rhythm conditions preserved not only a rhythm's accent coupling potential but also more tone duration pairings than did dissimilar thythm conditions. However, an explanation of the rhythmic similarity effect based strictly on the number of durational changes cannot suffice. There is evidence that the kind of temporal change (coupled vs. uncoupled) matters. First, differences in target accuracy due to differences in the number of durational changes were modest when accent coupling was held constant (similar rhythm vs, the original rhythm in Experiment 1). Second, across all rhythm conditions (Experiments 1 and $2 \mathrm{~A}$ ), performance should have declined with increases in the number of durational changes associated with a shift if this was critical; this did not happen. Thus, we conclude that an important component of rhythmic similarity effects on theme recognition involves accent coupling. In the context of novel rhythms, the time patterns that continue to temporally highlight a familiar theme are most likely to evoke a recognition response.

One general influence of placing melodies in novel rhythms may be that it encourages listeners to rely more on temporal relationships among prominent accents within a joint accent structure. If so, we might expect responses to decoys to show systematic effects of rhythmic similarity manipulations. They do. Rhythmic similarity not only supports better target recognition but it also boosts the chances that listeners will mistake a same decoy for a target. This finding confirms that of Jones et al. (1987). It is also consistent with a joint accent structure account of rhythmic similarity in music, which implies that the effective similarity of a durational pattern depends on the way in which it fits with a melodic one, given the context in which both are experienced.

The findings of Experiment $2 \mathrm{~A}$ are consistent with those of Experiment $\mathrm{I}$, in that listeners are better at differen- 
tiating targets from different decoys (as opposed to same decoys) in both rhythms. However, these trends are not so evident with less familiar melodies (Experiment 2B). Consequently, one might conclude that contour-pitch manipulations have minimal impact on listeners' memories of little-heard tunes, a conclusion consistent with other findings involving relatively long retention periods (Dowling \& Fujitani, 1971; Edworthy, 1985). However, in the present case, such a conclusion is premature.

In previous work, pitch contour has not been systematically manipulated as it has here, nor have rhythm and familiarity been manipulated jointly in long-term retention tasks. In the present case, there is evidence that these pitch contours are remembered for substantial time intervals. For example, listeners are better at differentiating little-heard targets from different decoys than from same decoys in the dissimilar rhythm conditions (mean $A_{g}$ is .665 with same decoys and .756 with different decoys). Although the overall interaction of melody with rhythm was not significant in this case, the difference is a sizable one that is buttressed by error rate analyses. Error rates indicate that these listeners judged the same decoys to be targets over $41 \%$ of the time in similar rhythm conditions and over $46 \%$ of the time in the dissimilar rhythm conditions; corresponding error rates to different decoys were substantially lower, hovering around $30 \%$ and $17 \%$ respectively! Thus, even after long retention intervals, a greater proportion of errors with less familiar tunes is due to contour-related confusions than is the case with familiar tunes. Although it is true that these errors indicate a biasing influence of higher order melodic structure, it is significant that this bias is specific to the melodic variation involved and that it persists over long time periods.

Nevertheless, a word of caution is appropriate in drawing conclusions about contour effects from such designs, because there is always the possibility that listeners respond to some distinctive pitch interval change(s) in one type of decoy (same or different). However, at least data from Experiment 1 indicate that under optimal conditions (original rhythm), the same and different decoys are not vastly different in discriminability, as might be expected if listeners relied solely on some distinctive interval difference to differentiate targets from decoys.

Finally, what about differential effects of rhythm on perceptual learning? Disproportionate benefits due to the similar rhythm condition are not evident early in learning. Instead, rhythm has a more limited early influence. However, rhythm and accent coupling clearly contribute to initial biases and ultimately help to shape perceptual learning. One scenario is that perceptual learning involves an early and crude responsiveness to highlighted pitch accents, and that with experience listeners abstract higher order time relations among these pitches. In this way, accent coupling may facilitate learning about "when" themebearing information occurs. If this interpretation is correct, recognition error rates for targets and different decoys in the similar rhythm conditions should decline with prac- tice, because in these cases listeners can reliably detect differentiating melodic information at attended-to serial locations. This is the case. This interpretation also implies that if target identification depends on abstraction of temporal relationships among highlighted contour-pitch accents, then error rates to same decoys in the similar rhythm will not decline with familiarization training. And they do not.

\section{GENERAL DISCUSSION AND CONCLUSIONS}

Listeners' sense of a familiar melodic theme after a substantial delay depends heavily upon the way a new rhythm highlights a melody's contour-related pitches. Particularly with fairly familiar tunes, reinstatement of rhythmic highlighting of some thematic information makes it more identifiable in both targets and decoys.

Perhaps these findings are not surprising. Intuitively, the same decoys represent lawful variations of the target melody's theme. But it is useful to discover that, depending on the rhythmic context, listeners mistake same decoys for targets over $40 \%$ of the time and, moreover, that these error rates do not decline as listeners become more familiar with the target. Together with findings of Jones et al. (1987), these data extend Dowling's (1978) hypothesis in showing that not only is contour-related information abstracted early in learning, but it is retained over substantial time periods.

As it was manipulated here, rhythm influenced not only the recognizability of a target melody but also the extent to which different variations of it (decoys) fooled listeners. Together, these findings imply that a pause-based encoding account of rhythm is too simple. With pause structure held constant, listeners are better at discriminating familiar targets from decoys when both appear in rhythms identical or similar to the original rhythm of the target. Performance depended on the serial loci of temporal accents within pause-based groups.

All novel rhythms take some toll on melody recognition; even changing a few durations within a pause-based group can hurt. Thus, target accuracy in Experiment 2 is somewhat poorer in the similar rhythm conditions than in the original rhythms (Experiment 1), even though pause segmentation and accent coupling are preserved in both. One reason stems from the fact that rearranging any duration within a rhythmic figure inevitably affects relative timing among all pitch accents (e.g., those from pitch skips etc.), not simply those involving contour change. The present design only manipulated the relative timing of pitch accents related to pitch contour; however, rhythmic shifts that modify timing of other pitch accents in a target should also lower its recognizability (Boltz \& Jones, 1986; Monahan et al., 1987).

These data also speak to the perceptual independence of melody and rhythm. Since absence of interactions is often taken as evidence for independence, it is clear that there is no simple solution to this issue. Evidence can be adduced for both independence (Experiment 2) and lack 
of it (Experiment 1), even with regard to the same dependent measure $\left(A_{g}\right)$. Furthermore, on another level, it is also evident that in these tasks listeners who were explicitly instructed to ignore rhythm and pay attention only to pitch changes simply could not do this. The main effect of rhythm on recognition of melodies with identical pause punctuations, as indexed by all dependent measures, tells us how difficult it is for average listeners to focus on melodic information independently of rhythm. Perhaps the most plausible approach is one that maintains that presence or absence of interactions depends both on the way melodic and rhythmic structures fit together to determine a joint accent structure and on how listeners abstract and use its relational properties within some larger context.

Finally, these findings have been interpreted in terms of the way temporal context, summarized by joint accent structure, guides attending. With practice, coupled accents within an accent-time hierarchy come to command attending to temporal loci of nonadjacent events (Jones, 1985, 1987; Jones \& Boltz, 1989). Others also address temporal context effects, but do so with clock models where various accents are assumed to occur with fixed time periods (e.g., Povel \& Essens, 1985; Monahan et al., 1987).

However an alternative, noncontextual, account of rhythmic influences is possible. It argues that coupling "works" because temporal accents offer more time to process pitch information (Idson \& Massaro, 1976; Povel \& Okkerman, 1981). Although this may be part of the story, it is unlikely to be the whole story, because all time intervals associated with pitches are above thresholds suggested for improvements in pitch recognition with time (i.e., $70-250 \mathrm{msec}$ ).

\section{REFERENCES}

BAMBER, C. (1975). The area above the ordinal dominance graph and the area below the receiver operating characteristic graph. Journal of Mathematical Psychology, 12, 387-415.

BARTLETt, J. C., Dowling, W. J. (1980). The recognition of transposed melodies: A key-distance effect in developmental perspective. Joumal of Experimental Psychology: Human Perception \& Performance, 6, 501-515.

Boltz, M. JoNes, M. R. (1986). Does rule recursion make melodies easier to reproduce? If not, what does? Cognitive Psychology, 18, 389-431.

Burbidge, S., Jones, M. R. (1982). The function of rule-governed serial contexts in recognition of embedded tones. Psychomusicology, 2, 47-63.

Butler, D., \& Brown, H. (1984). Tonal structure versus function: Studies of the recognition of harmonic motion. Music Perception, 2, 6-24.

Davison, T. C. B., \& JAGACINSKI, R. J. (1977). Nonparametric analysis of signal detection confidence ratings. Behavior Research Methods \& Instrumentation, 9, 545-546.

DeLIEGE, I. (1987). Grouping conditions in listening to music: An approach to Lerdahl \& Jackendoff's grouping preference rules. Music Perception, 4, 325-359.

Deutsch, D. (1980). The processing of structured and unstructured tonal sequences. Perception \& Psychophysics, 28, 381-389.

Dowling, W. J. (1973). Rhythmic groups and subjective chunks in memory for melodies. Perception \& Psychophysics, 14, 37-40.

DowLING, W. J. (1978). Scale and contour: Two components of a theory of memory for melodies. Psychological Review, 85, 341-354

Dowling, W. J., BARLETT, J. C. (1981). The importance of inter- val information in long-term memory for melodies. Psychomusicology, $1,30-49$.

Dowling, W. J., Fujitani, D. S. (1971). Contour, interval, and pitch recognition in memory for melodies. Joumal of the Acoustical Society of America, 49,524-531.

Dowling, W. J., Harwood, D. L. (1986). Music Cognition. New York: Academic Press

Drson, M. C., W Watkins, A. J. (1984). A figural approach to the role of melodic contour in melody recognition. Perception \& Psychophysics, 35, 477-488.

EDWorthy, J. (1985). Melodic contour and musical structure. In P. Howell, I. Cross, \& R. West (Eds.), Musical structure and cognition (pp. 169-188). New York: Academic Press.

Handel, S. (1984). Using polyrhythms to study rhythm. Music Perception, 1, 465-484

HANDEL, S. (1989). Listening: An introduction to perception of auditory events. Cambridge, MA: MIT Press

Idson, W. L., MAssaro, D. W. (1976). Cross-octave masking of single tones and musical sequences: The effects of structure on auditory recognition. Perception \& Psychophysics, 19, 155-175.

JoNES, M. R. (1976). Time, our lost dimension: Toward a new theory of perception, attention, and memory. Psychological Review, 83, 323-355

JONEs, M. R. (1981). Music as a stimulus for psychological motion: Par I. Some determinants of expectancies. Psychomusicology, 1 , 34-51.

JONES, M. R. (1982). Music as a stimulus for psychological motion: Part II. An expectancy model. Psychomusicology, 2, 1-13.

JONES, M. R. (1985). Structural organization of events in time. In J. Michon \& J. Jackson-Roy (Eds.), Time, mind, and behavior (pp. 192-214). Heidelberg: Springer-Verlag.

JONES, M. R. (1987). Dynamic pattern structure in music: Recent theory and research. Perception \& Psychophysics, 41, 621-634.

JONES, M. R. (1990). Musical events and models of musical time. In A. Block (Ed.), Cognitive models of psychological time (pp. 207-240). Hillsdale, NJ: Erlbaum.

JONES, M. R., Boltz, M. (1989). Dynamic attending and responses to time. Psychological Review, 96, 459-491.

JoNes, M. R., BoltZ, M. , KIDD, G. (1982). Controlled attending as a function of melodic and temporal context. Perception \& Psychophysics, 32, 211-218

Jones, M. R., Summerell, L., MArshburn, E. (1987). Recognizing melodies: A dynamic interpretation. Quarterly Journal of Experimental Psychology, 39, 89-121.

MARTIN, J. G. (1972). Rhythmic (hierarchical) versus serial structure in speech and other behavior. Psychological Review, 79, 487-509.

Monahan, C., Carterette, E. C. (1985). Pitch and duration as determinants of musical space. Music Perception, 3, 1-32.

Monahan, C. B., Kendall, R. A., Carterette, E. C. (1987). The effect of melodic and temporal contour on recognition memory for pitch change. Perception \& Psychophysics, 41, 576-600.

Palmer, C., \& Krumhansl, C. L. (1987a). Independent temporal and pitch structures in determination of musical phrases. Journal of $E x$ perimental Psychology: Human Perception \& Performance, 13, $116-126$

Palmer, C., Krumhansl, C. L. (1987b). Pitch and temporal contributions to musical phrase perception: Effects of harmony, performance timing, and familiarity. Perception \& Psychophysics, 41. 505-518

Pollack, I., Norman, D. A., \& Galanter, E. (1964). An efficient non-parametric analysis of recognition memory. Psychonomic Science, $1,327-328$

Povel, D.-J., \& Essens, P. (1985). Perception of temporal patterns. Music Perception, 2, 411-440.

Povel, D.-J., OKKERMaN, H. (1981). Accents in equitone sequences. Perception \& Psychophysics, 30, 565-572.

Thomassen, J. M. (1982). Melodic accent: Experiments and a tentative model. Journal of the Acoustical Society of America, 71, 1596-1605

WaLlace, W. T., Rubin, D. C. (in press). Characteristics and constraints in ballads and their effects on memory. Discourse Processes. 
WatKINS, A. J. (1985). Scale, key, and contour in the discrimination of tuned and mistuned approximations to melody. Perception \& Psychophysics, 37, 275-285.

YESTON, M. (1976). The stratification of musical rhythm. New Haven, CT: Yale University Press.

\section{NOTES}

1. A musical key in tonal Western music refers to a diatonic set of seven pitches that conforms to constraints on successive pitch intervals of the form 2ST, 2ST, 1ST, 2ST, 2ST, 2ST, 1ST, where ST refers to a semitone pitch interval. The semitone is the smallest pitch division of the octave in Western music; it is logarithmically defined as a constant ratio between two frequencies of $1.059 .$. according to equal temperament tuning. Melodic structure in tonal music builds on special diatonic relationships; among these are ones involving the psychological anchoring function of the keynote or tonic (e.g., the tonic is $\mathrm{C}$ in the C major scale, which consists of C D E F G A B C').
2. Some have used the term pitch inflection to refer to accents associated with pitch contour (Monahan et al., 1987). Their use differs from the present definition of contour-pitch accents in two ways: (1) They imply an interval-plus-pitch direction change; and (2) they do not conceive of differential accent weights as a function of local versus global pitch peaks.

3. If central pitches themselves are taken as the referent for establishing interval time relations among the contour-pitch accents, then some different decoys actually change the temporal order of these accents as they appear in their targets. However, the general rationale involved changing the relative time of prominent central pitches relative to the fixed openings segment. This is most accurately formalized as a $\pm \Delta t$ change between two tone onsets. In terms of a joint accent structure analysis, each pitch is assigned an accent weight, which in turn defines its level in an accent/time hierarchy. More prominent central pitches contribute to heavier accent weights, which are, in turn, correlated with longer time intervals in this analysis (Jones, 1976, 1987). Thus, changing the serial location of any pitch can also modify relative time properties of an accent/time hierarchy (Jones, 1990).

Appendix

Stimuli Used in All Experiments

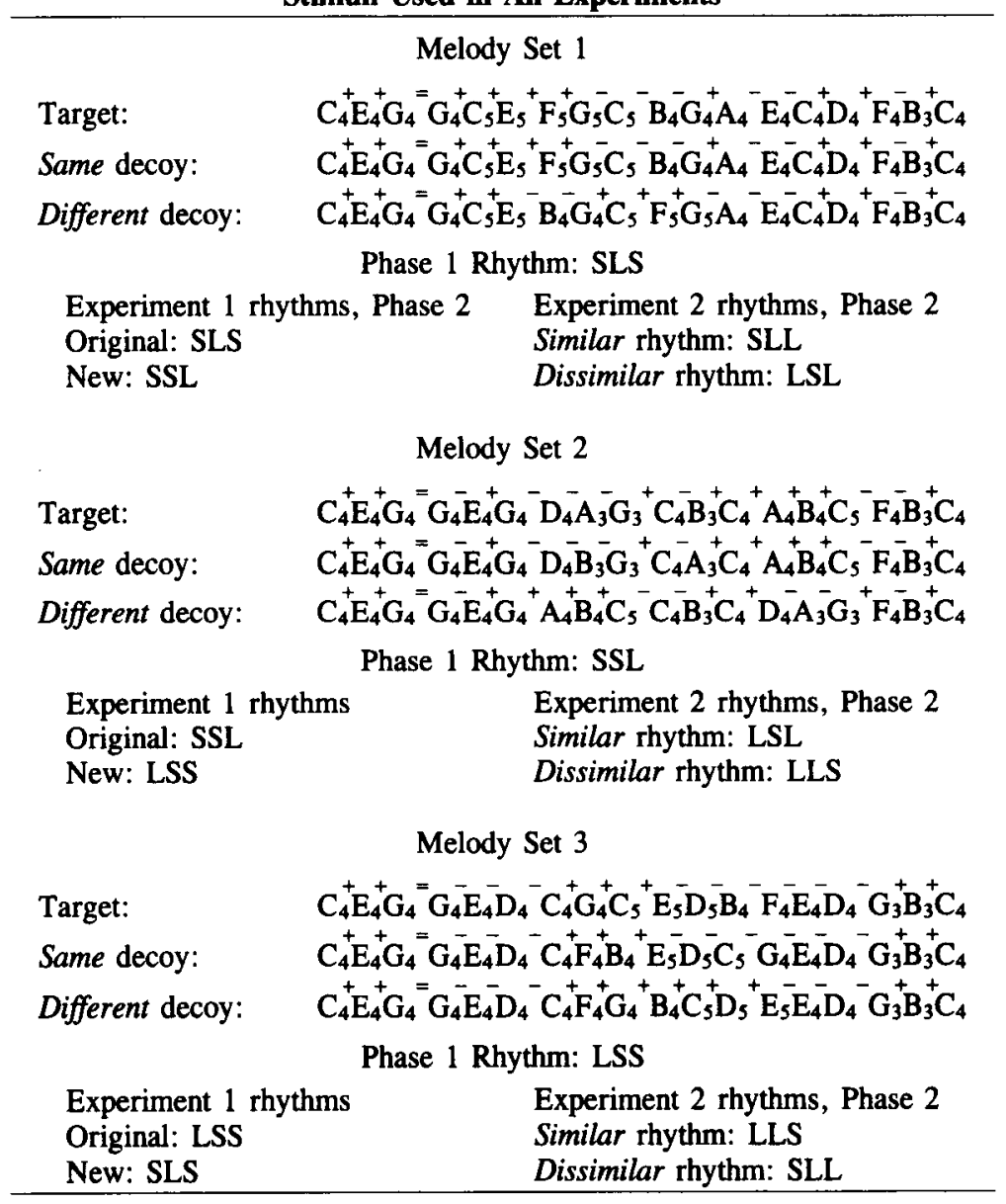

(Manuscript received June 15, 1989;

revision accepted for publication May 8, 1990.) 